\title{
Boundary bound states in integrable quantum field theory
}

\author{
E. Corrigan* \\ Department of Mathematics, \\ University of York, \\ York YO10 5DD, UK \\ and \\ Department of Mathematical Sciences, \\ University of Durham, \\ Durham DH1 3LE, UK
}

ABSTRACT: The purpose of this talk is to sketch some recent progress which has been made in calculating non-perturbatively the reflection factors for the sinh-Gordon model restricted to a half-line by integrable boundary conditions. The essential idea is to calculate the energy spectrum of boundary breathers in two independent ways; firstly by using the boundary bootstrap and secondly by quantizing the classical solutions corresponding to boundary breathers. Comparing these two calculations provides a way to determine the dependence of the reflection factors on the parameters introduced into the Lagrangian by the boundary conditions. The basic idea is illustrated using a massive free scalar field with a linear boundary condition confining it to a half-line.

\section{Introduction}

Although boundary conditions are extremely important for field theory - think of monopoles or vortices, for example, or instantons - the study of integrable quantum field theory restricted to a half-line or an interval by integrable boundary conditions is not yet fully developed. In particular, many interesting areas are yet to be explored and the foremost of these is the classification of the reflection factors which determine the manner in which a particle rebounds from a boundary. In 1993 Ghoshal and Zamolodchikov [1 $118^{1}, 119$ ignited activity in this subject by analysing the possible behaviour of the sine-Gordon model restricted to a half-line. They discovered a twoparameter family of boundary conditions which preserved integrability, in the sense of preserving after suitable modification all the energy-like conserved quantities, while violating those which

\footnotetext{
*E-mail: ec9@york.ac.uk
}

were momentum-like. Clearly, the same facts would be true of the sinh-Gordon model, which is even simpler in the sense of having a particle spectrum consisting of a single scalar particle and nothing else. The reflection factors for the states belonging to the sine-Gordon particle spectrum (solitons, anti-solitons and breathers) were computed although the relationship between two parameters appearing in the reflection factors and the two parameters appearing in the classical Lagrangian specifying the boundary conditions remained largely obscure. The purpose of developing the techniques reviewed here was to remove this obscurity. It seems such a simple matter that it is surprising that it has taken so many years to find a way to resolve it.

Apart from the troubles relating Lagrangian parameters to the parameters appearing in reflection factors there are other mysteries too. For example, the sinh-Gordon model is the simplest of the affine Toda field theories and yet it is the only 
one in the ade series of cases with a set of boundary conditions with free parameters. For all the rest, the possible integrability-preserving boundary conditions unexpectedly form a discrete set

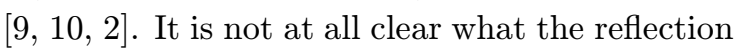
factors are which correspond to each member of this discrete set, although a variety of reflection factors have been found using algebraic means (see for example [14ind). Our long-term goal is to find a complete classification and a full understanding of the curious pattern of integrable boundary conditions discovered in [izi].

For a review of the background to some of these questions see [1] $\left.\overline{1}_{1}^{1}\right]$.

\section{A free scalar field with a linear boundary condition}

In this section, to set the scene, consider a free massive scalar field confined to the left half-line $(x<0)$ by a linear boundary condition at $x=0$. Its equation of motion and boundary condition are:

$$
\left(\partial^{2}+m^{2}\right) \phi=0, x<0 ; \partial_{x} \phi=-\lambda \phi, x=0 .
$$

Clearly, there is a set of solutions to (2.1i) corresponding to a superposition of left and rightmoving waves of the form

$$
\phi=e^{-i \omega t}\left(e^{i k x}+R_{0}(k) e^{-i k x}\right)+c . c .
$$

with $w^{2}=m^{2}+k^{2}$ and a 'reflection factor' given by

$$
R_{0}=\frac{i k+\lambda}{i k-\lambda} .
$$

If $\lambda<0$ there is also a periodic solution of the form (whose existence is indicated by a pole in $R_{0}$ at $\left.k=-i \lambda\right)$,

$$
\phi=A \cos \omega t e^{-\lambda x}, \omega^{2}=m^{2}-\lambda^{2},
$$

provided also $0>\lambda>-m$. Clearly, this solution is slightly delicate and fails to exist at all for a massless free field. It is a harmonic oscillator 'glued' to the boundary. Its energy is computed to be $\mathcal{E}$ where

$$
\begin{gathered}
\mathcal{E}=\int_{-\infty}^{0} d x\left(\dot{\phi}^{2}+\phi^{\prime 2}+m^{2} \phi^{2}\right)+\lambda \phi^{2}(0, t) \\
=-\frac{\omega^{2} A^{2}}{2 \lambda}
\end{gathered}
$$

Quantizing this periodic system leads to a tower of states with energies $(n+1 / 2) \omega$.

On the other hand, in the context of integrable field theory, we know that the S-matrix for the free massive particle is unity and the reflection factor is identical to the classical version $(2.3)$. That is, setting $\omega=m \cosh \theta, k=$ $m \sinh \theta$ we have the relationship between 'in' and 'out' states

$$
\left|\theta>_{\text {out }}=R_{0}(k)\right|-\theta>_{\text {in }}
$$

where 'in' refers to a particle of rapidity $\theta$ approaching the boundary and 'out' refers to the same particle at a later time after reflecting from the boundary. The pole in $R_{0}$ at $k=-i \lambda$ indicates a boundary bound state at an imaginary rapidity $\left(\theta=i \psi=-i \sin ^{-1}(\lambda / m)\right)$ and the boundary bootstrap $[1 \overline{1}, \overline{1}, \overline{1}, \overline{9}$, , indicates that the energy of the excited boundary is given by

$$
\mathcal{E}_{1}=\mathcal{E}_{0}+m \cos \psi=\mathcal{E}_{0}+\omega,
$$

with an associated reflection factor again equal to $R_{0}$. The reason for this is that the reflection factor $R_{1}$ for the particle rebounding from the excited boundary is given by

$$
R_{1}(\theta)=S(\theta-i \psi) R_{0}(\theta) S(\theta+i \psi)
$$

and the S-matrix is unity. Repeating the process leads to the tower of harmonic oscillator states.

So, the basic idea which we wished to exploit was to follow this line of reasoning in the sinhGordon model, constructing a tower of boundary states in two different ways: firstly, using the formal bootstrap, and secondly, using a direct quantization of periodic solutions corresponding to an 'oscillator' attached to the boundary. It is expected that the same approach will be applicable to the other Toda theories as well.

\section{The sinh-Gordon model}

We begin by establishing notation and reminding ourselves of certain relevant facts about the sinhGordon model on a half-line. Its field equation and integrable boundary conditions are

$$
\begin{aligned}
& \partial^{2} \phi=-\frac{\sqrt{8} m^{2}}{\beta^{2}} \sinh (\sqrt{2} \beta \phi), x<0 \\
& \partial_{x} \phi=\frac{\sqrt{2} m}{\beta}\left(\epsilon_{0} e^{-\beta \phi / \sqrt{2}}-\epsilon_{1} e^{\beta \phi / \sqrt{2}}\right), x=0,
\end{aligned}
$$


where $m$ is a mass parameter (which we shall set to unity), $\beta$ is the bulk coupling, and $\epsilon_{0}$ and $\epsilon_{1}$ are the two parameters introduced by the boundary conditions. Notice that when $\epsilon_{0} \neq \epsilon_{1}$ the bulk symmetry under the transformation $\phi \rightarrow$ $-\phi$ is lost. Although it is not strictly necessary to assume that $\left|\epsilon_{i}\right| \leq 1$, in what follows it will often be useful to put

$$
\epsilon_{i}=\cos a_{i} \pi, i=0,1, \quad 0 \leq a_{1} \leq a_{0} \leq 1 .
$$

The basic question is to give a complete characterization of the reflection factors as functions of the bulk coupling and the two boundary parameters.

The sinh-Gordon model is not free but its S-matrix is well-known and may be written compactly as follows [1] $\left.{ }_{1}^{1} \bar{n}_{1}\right]$

$$
S\left(\theta_{12}\right)=-\frac{1}{(B)(2-B)}, B(\beta)=\frac{\beta^{2} / 2 \pi}{1+\beta^{2} / 4 \pi},
$$

using the block notation,

$$
(x)=\frac{\sinh \left(\theta_{12} / 2+i \pi x / 4\right)}{\sinh \left(\theta_{12} / 2-i \pi x / 4\right)},
$$

introduced in $[3 \overline{3}]$. The S-matrix is invariant under the transformation $\beta \rightarrow 4 \pi / \beta$.

The reflection factors may be deduced from those computed by Ghoshal [1 $\left.1 \overline{9}_{1}^{\prime}\right]$ for the sineGordon model, and have the form

$$
R(\theta)=\frac{(1)(1+B / 2)(2-B / 2)}{(1-E)(1+E)(1-F)(1+F)},
$$

where $E, F$ are two functions which are independent of the rapidity but depend upon the bulk coupling and the boundary coupling parameters in a manner which needs to be determined. The classical limit of $(\overline{3} . \overline{5})$ as $\beta \rightarrow 0$ has been calculated independently [1] $a_{0}+a_{1}, F \rightarrow a_{0}-a_{1}$, using the notation (3.21). Indeed, (3.5i) is the simplest guess having the appropriate classical limit and satisfying the 'crossing unitarity relation' [1] $\left.\overline{1}_{-1}^{\prime}, \overline{1} \overline{6}_{1}^{\prime \prime}\right]$

$$
R(\theta+i \pi / 2) R(\theta-i \pi / 2) S(2 \theta)=1 .
$$

We are also intrigued to know what happens to the weak-strong coupling invariance enjoyed by the S-matrix factor.

The expression (3.5ils $)$ has also been checked

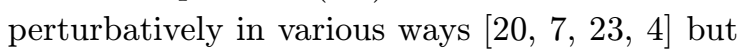
the details will not be described here.

\section{Using the boundary bootstrap}

Examining the expression $(3.5)$ reveals that it may have poles at $\theta=i \psi, 0<\psi<\pi / 2$ and that these poles cannot come from the numerator factors, only from zeroes of the denominator. Moreover, the existence of suitable poles depends on the value of $E$ or $F$. For example, with $1<E<2$ there are poles at $\theta=i(E-1) \pi / 2$. What is more, knowing the classical limit of $E$ and $F$ is useful because it informs us that it is plausible, given the ranges of $a_{0}$ and $a_{1}$, that $E$ and $F$ do not lie simultaneously in the range $(1,2)$. This fact is true for the classical limit, and the order $\beta^{2}$ perturbative calculations do not upset it. So, we shall proceed as though it were true and see that the resulting expressions for $E$ and $F$ are consistent with it. For the symmetric case, $\left(a_{0}=a_{1}\right), F=0$, and the question does not even arise. More details of these calculations may be found in [is] and [1 $[\overline{1} 2]$.

Then, provided $1<E<2$ we may compute a tower of states using the boundary bootstrap (2.7). The result is a sequence of states whose energies are given by

$$
\mathcal{E}_{n+1}=\mathcal{E}_{n}+m \cos \frac{\pi}{2}(n B-E+1),
$$

and for which the associated reflection factors have the form

$$
\begin{aligned}
& R_{n}(\theta)=R_{D} \frac{(1+E+B)(1-E-B)}{(1-E+n B)(1+E-n B)} \\
& \times \frac{1}{(1-E+(n-1) B)(1+E-(n-1) B)},
\end{aligned}
$$

where

$$
R_{D}=\frac{(1)(1+B / 2)(2-B / 2)}{(1-F)(1+F)}
$$

is $n$-independent and never contains contributing poles.

Notice that the number of new types of pole is limited because, even if $E$ is initially within the appropriate range, eventually $E-1-n B$ will move out of range. It seems natural to interpret the pole at $i(E-1-(n-1) B) \pi / 2$ as a 'crossed' process in which the state with energy 
$\mathcal{E}_{n}$ drops down to the state at energy $\mathcal{E}_{n-1} \cdot{ }^{1}$ If we interpreted this pole as a new state then the reflection factor corresponding to it would contain higher order poles and these in turn would need to be explained.

The next part of the argument requires a set of classical solutions to the system of equations (3.1).

\section{Classical boundary breathers}

The classical periodic solutions which we shall use may all be described within Hirota's ansatz. That is, for our purposes it is convenient to set

$$
\phi=-\frac{\sqrt{2}}{\beta} \ln \frac{\tau_{0}}{\tau_{1}},
$$

where

$$
\begin{aligned}
& \tau_{a}=1+(-)^{a}\left(E_{1}+E_{2}+E_{3}\right)+A_{12} E_{1} E_{2} \\
& \quad+A_{13} E_{1} E_{3}+A_{23} E_{2} E_{3}+A_{12} A_{13} A_{23} E_{1} E_{2} E_{3},
\end{aligned}
$$

and

$$
\begin{gathered}
E_{p}=e^{a_{p} x+b_{p} t+c_{p}} \\
a_{p}=2 \cosh \rho_{p}, b_{p}=2 \sinh \rho_{p} \\
A_{p q}=\tanh ^{2}\left(\frac{\rho_{p}-\rho_{q}}{2}\right) .
\end{gathered}
$$

At this stage, the constants $c_{\rho}$ are not determined.

On the whole line, these solutions are not particularly interesting because for each $t$ they develop a singularity at some $x$. Moreover, owing to the singularities their total energy will be infinite. However, we are interested in periodic solutions defined only on the region $x<0$ and therefore it is possible that solutions satisfying

\footnotetext{
${ }^{1}$ However, there is a bit of a mystery, or at least an inconsistency of interpretation between this case and the free field case. Here, we have in mind that there ought to be a finite number of boundary states just as there are a finite number of sine-Gordon breathers at most, at a given bulk coupling (see [22] for a review); only in the classical limit do we expect to have infinitely many bound states. On the other hand, it seems natural to have the infinite tower when we are discussing the free field boundary states. Thanks to Patrick Dorey for a discussion on this issue.
}

the boundary conditions can be non-singular except at points beyond the boundary; in effect, the singularities are hidden away in the region $x>0$. This hope turns out to be realised rather neatly and the details are given more fully in [i8]. The trick is to select $E_{1}$ and $E_{2}$ to be time dependent and periodic (ie, inevitably complex), and to satisfy $E_{1}^{*}=E_{2}$, while taking $E_{3}$ to be real. Then, the solution given via (5.1) is real and the boundary conditions can be satisfied.

For the particular case where $\epsilon_{0}=\epsilon_{1} \equiv \epsilon$, it is enough to set $E_{3}=0$ and we have,

$$
\begin{aligned}
\tau_{a}=1 & +\frac{(-)^{a}}{\tan \rho} e^{2 x \cos \rho} \cos (2 t \sin \rho) \sqrt{\frac{\epsilon+\cos \rho}{\epsilon-\cos \rho}} \\
& -e^{4 x \cos \rho} \frac{\epsilon+\cos \rho}{\epsilon-\cos \rho} .
\end{aligned}
$$

The expression ( $\left(\begin{array}{l}5 \\ 5\end{array}\right.$ $\operatorname{riod} \pi / \sin \rho$. However, for the corresponding solution constructed via (15.1) to be non-singular, $\epsilon$ and $\rho$ must be constrained as follows:

$$
-1<\epsilon<0, \quad \cos \rho \leq-\epsilon .
$$

As expected, $\epsilon$ should be negative (meaning that there is a competition between the different signs of the boundary and bulk contributions to the energy), but not too negative (cf the free field case). More of a surprise is the constraint on $\rho$ since it means that there is a minimum frequency for the breather but, at that minimum frequency the amplitude of the breather has shrunk to zero. This behaviour is different to the behaviour of the sine-Gordon breathers since for those the frequency drops to zero as the amplitude collapses to zero. It seems the boundary breathers are still breathing faintly despite their collapse when $\cos \rho=-\epsilon$. This behaviour is reminiscent of a standard oscillator whose amplitude may be tuned to zero independently of its frequency. In a nonlinear system for a given boundary condition the frequency governs the amplitude of the periodic solution.

In the general case, the solutions are substantially more complicated. The boundary conditions require

$$
\begin{aligned}
e^{c_{3}} & =\frac{r}{q^{2}}, e^{c_{1}}=e^{c_{2}}=\frac{s}{\tan \rho}, r=\frac{q_{-}}{q_{+}} \\
s^{2} & =\frac{1}{q^{2}} \frac{1-q^{2} q_{+}^{2}}{1-q^{-2} q_{+}^{2}} \frac{1-q^{-2} q_{-}^{2}}{1-q^{2} q_{-}^{2}}
\end{aligned}
$$


where

$$
q=\tan \frac{\rho}{2}, q_{ \pm}=\tan \frac{\pi}{4}\left(a_{0} \pm a_{1}\right) .
$$

The general boundary breathers have no singularities in the region $x<0$ provided

$$
\begin{gathered}
\cos \frac{\pi}{2}\left(a_{0}+a_{1}\right)<0, \cos \frac{\pi}{2}\left(a_{0}-a_{1}\right)>0 \\
0<\cos \rho<-\cos \frac{\pi}{2}\left(a_{0}+a_{1}\right) .
\end{gathered}
$$

The restrictions (15.8) were found numerically using Maple assuming that the parameters $a_{0}$ and $a_{1}$ lie in the range indicated in (3.2). The energy of the general periodic solution given by the data $(15.6)$ is given by [i]

$\mathcal{E}_{b}=-\frac{8}{\beta^{2}}\left(1+\cos \rho-\frac{1}{2}\left(\sin \frac{\pi a_{0}}{2}+\sin \frac{\pi a_{1}}{2}\right)^{2}\right)$.

\section{Semi-classical quantization}

Now that we have a collection of periodic solutions the next step is to quantize them to determine their energy spectrum. We shall do this by adapting the WKB methods proposed long ago by Dashen, Hasslacher and Neveu [1] $\overline{3}$. Here it will be enough to outline the prescription and more of the details are to be found in $\left[\begin{array}{ll}1 & 1 \\ 1 & 12\end{array}\right]$. The procedure is certainly non-perturbative but we have no way of telling at present if it is also exact. In the case of the sine-Gordon model in the bulk, the WKB methods do give exact results in agreement with calculations made within the eight vertex model (for example, see $2 \overline{1} \overline{1}$ ). It remains to be seen whether this feature will persist in the presence of boundaries. As we shall see, there are several surprises which render the analysis for the general periodic solution manageable.

The first ingredient we need is the classical action integrate over a single period of the boundary breathers. Since we know the energy already, it will be necessary to calculate the appropriate integral of $\dot{\phi}^{2}$. Thus,

$$
\begin{aligned}
S_{\text {class }} & =\int_{0}^{T} d t \int_{-\infty}^{0} d x \dot{\phi}^{2}-T \mathcal{E}_{b} \\
& =\frac{8 \pi}{\beta^{2}}\left(\rho-\pi\left(1-\frac{a_{0}+a_{1}}{2}\right)\right)-T \mathcal{E}_{b}
\end{aligned}
$$

This is a remarkable result because the first part of the expression depends only on the combination $a_{0}+a_{1}$. For the symmetric case $a_{0}=a_{1}$, the expression for the action is relatively straightforward to compute analytically by a suitable sequence of changes of integration variables. However, in the general case the result was deduced numerically and the rather convincing numerical evidence we have available is summarised in the appendix to [1 $12_{2}^{\prime}$. It would be nice to have an analytic derivation of $(\underline{6} \cdot \overline{1} \overline{1})$, however it has eluded us so far.

Besides the classical action we shall also need the linear perturbations around the breathers regarded as a background. In other words put $\phi=\phi_{0}+\eta$, where $\phi_{0}$ is a breather and solve the following linear equations for $\eta$ :

$$
\begin{aligned}
& \partial_{t}^{2} \eta-\partial_{x}^{2} \eta+4 \eta \cosh \left(\sqrt{2} \beta \phi_{0}\right)=0, x<0 \\
& \partial_{x} \eta+\left(\epsilon_{0} e^{-\beta \phi_{0} / \sqrt{2}}+\epsilon_{1} e^{\beta \phi_{0} / \sqrt{2}}\right)=0, x=0 .
\end{aligned}
$$

The solutions in which we are interested are asymptotically plane-wave solutions, which means that as $x \rightarrow-\infty$ they have the form,

$$
\eta \sim e^{-i \omega t}\left(e^{i k x}+R(k) e^{-i k x}\right), \omega^{2}=k^{2}+4,
$$

where $R$ is the classical reflection factor in the breather background. Actually, we shall need the classical reflection factor in the classical ground state background as well (this particular reflection factor, incidentally, is the classical limit of Ghoshal's formula (3.5int). Fortunately, a further use of Hirota's method, adding two infinitesimal exponentials to (5.2) in the standard manner, allows a straightforward derivation of the reflection factors we need. They are,

$$
\begin{aligned}
R_{\text {breather }}= & \left(\frac{i k-2 \cos \rho}{i k+2 \cos \rho}\right)^{2} \frac{i k-2}{i k+2} \\
& \times \frac{i k-2 \cos a_{+}}{i k+2 \cos a_{+}} \frac{i k+2 \cos a_{-}}{i k-2 \cos a_{-}} \\
R_{\text {ground }}= & \frac{i k-2}{i k+2} \frac{i k+2 \cos a_{+}}{i k-2 \cos a_{+}} \frac{i k+2 \cos a_{-}}{i k-2 \cos a_{-}}
\end{aligned}
$$

and, for notational convenience it is useful to define $a_{ \pm}=\pi\left(a_{0} \pm a_{1}\right) / 2$. The second of the reflection factors given in (6.4) allows us to deduce 
the classical limit for $E$ or $F$ quoted earlier. Another surprising feature which we did not expect is that the ratio of the two classical reflection factors does not depend on the combination $a_{0}-a_{1}$.

The linear perturbations of the breathers do not have the same periodicity as the breathers themselves. Rather, their frequencies provide the modification to the action which is to be used in the WKB aproximation. Thus, we need to calculate a quantity $\Delta$ defined by

$$
\Delta=\frac{T}{2} \sum\left(\omega_{\text {breather }}-\omega_{\text {ground }}\right),
$$

and, in terms of this the quantum action is defined to be

$$
S_{\mathrm{qu}}=S_{\text {class }}-\Delta .
$$

The evaluation of $\Delta$ is not quite straightforward although we are fortunate to have the old work of Dashen, Hasslacher and Neveu as a guide. The method we adopted runs as follows. First discretize the sum over the frequencies and then regulate it by removing an infinite piece. It is convenient to use a Dirichlet boundary condition at $x=-L$, so that $\eta(-L, t)=0$, effectively placing the system in a box without changing the boundary condition at $x=0$. Subsequently, the limit $L \rightarrow \infty$ will be taken at the end of the calculation. Removing the infinite part of the discrete sum corresponds to making a choice of normalordering in a perturbative approach to the field theory.

Once the Dirichlet condition is imposed the possible discrete values of the parameters $k$ appearing in $(\underline{6} . \overline{3}-\overline{3})$ are given by

$$
e^{-2 i L k_{B}}=R_{B}\left(k_{B}\right), e^{-2 i L k_{0}}=R_{0}\left(k_{0}\right),
$$

where the subscripts $B$ and 0 refer to the breather and ground state, respectively. Typically, (6.7) have infinitely many real solutions together with a small number of purely imaginary solutions. The latter do play a rôle which will not be discussed here. The details may be found in [1 12 2']. For large $L$, it is useful to note that the difference between $k_{B}$ and $k_{0}$ is small, prompting us to write for the solutions to 6.71$)$

$$
\left(k_{B}\right)_{n}=\left(k_{0}\right)_{n}+\frac{1}{L} \kappa\left(\left(k_{0}\right)_{n}\right),
$$

where the function $\kappa$ satisfies

$$
e^{2 i \kappa(k)}=\left(\frac{i k+2 \cos \rho}{i k-2 \cos \rho} \frac{i k+2 \cos a_{+}}{i k-2 \cos a_{+}}\right)^{2} .
$$

Again, we notice the curious and surprising fact that only the combination $a_{0}+a_{1}$ appears.

The detailed calculation of $\Delta$ may be found

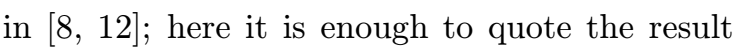
which is pleasingly simple:

$$
\begin{array}{r}
\Delta=\pi-\frac{2}{\sin \rho}\left(\cos \rho+\cos a_{+}+\rho \sin \rho\right. \\
\left.+\left(a_{+}-\pi / 2\right) \sin a_{+}\right) .(6
\end{array}
$$

The WKB prescription instructs us to define $\mathcal{E}_{\mathrm{qu}}=-\partial S_{\mathrm{qu}} / \partial T$, and then set

$$
S_{\mathrm{qu}}+T \mathcal{E}_{\mathrm{qu}}=2 n \pi
$$

where $n$ is a (positive) integer, or zero. In other words, assembling all the ingredients, we have the quantization rule

$$
2 n \pi=\frac{4}{B}(\rho-\pi / 2)+\frac{8 \pi}{\beta^{2}}\left(a_{+}-\pi / 2\right),
$$

from which we deduce a set of angles $\rho_{n}$, and thence a tower of energies $\mathcal{E}_{n}$ satisfying

$$
\begin{aligned}
& \mathcal{E}_{n+1}=\mathcal{E}_{n}+\frac{8}{\pi B} \sin \frac{\pi B}{4} \times \\
& \cos \frac{\pi}{2}\left(\frac{4 B}{\beta^{2}}\left(a_{+}-\pi / 2\right)-(n+1 / 2) B\right),
\end{aligned}
$$

which should be compared with (4.1.1). Before making the comparison it is perhaps worth noting that as $\beta \rightarrow 0$

$$
\rho_{n} \rightarrow\left(\pi-a_{+}\right), \mathcal{E}_{n} \rightarrow(n+1 / 2) \omega_{0},
$$

where $\omega_{0}=2 \sin a_{+}$is the lowest possible breather frequency.

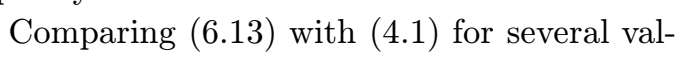
ues of $n$ yields two pieces of information. First of all the mass of the sinh-Gordon particle must be given by

$$
m(\beta)=\frac{8}{\pi B} \sin \frac{\pi B}{4},
$$

and, secondly, the parameter $E$ appearing in (3.5) for the reflection factor must be related to the boundary parameters and the bulk coupling by

$$
E\left(\beta, a_{0}, a_{1}\right)=\left(a_{0}+a_{1}\right)(1-B / 2) .
$$


This expression agrees perfectly with the classical limit as $\beta \rightarrow 0$, and with the result given by Ghoshal and Zamolodchikov for the Neumann boundary condition. There, $a_{0}=a_{1}=1 / 2$ and $E=1-B / 2$ as they claimed. The expression

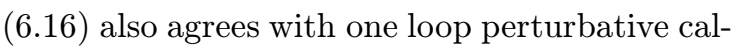
culations [17', [23, and with the known results at

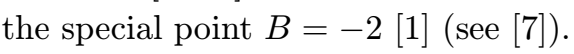

As far as the other parameter $F$ is concerned we know its classical limit, and we also know its expansion to order $\beta^{2}$, at least to first order in the difference $a_{0}-a_{1}$ (see $[\overline{4} \bar{i} \mid)$. Unfortunately, when the bulk $Z_{2}$ symmetry is broken the perturbation calculations are substantially more complicated, and indeed unfinished for arbitrary $a_{0}, a_{1}$ (see [is with the expression

$$
F\left(\beta, a_{0}, a_{1}\right)=\left(a_{0}-a_{1}\right)(1-B / 2) .
$$

In any case, had we chosen to replace $a_{1}$, say, by $-a_{1}$, we would expect $E$ and $F$ to interchange (since $E$ by itself is not invariant under such a change).

Notice that since $0 \leq 1-B / 2 \leq 1$, the assumptions we made concerning the regions in the parameter space for which the reflection factor (13.5) has poles are vindicated.

One slight criticism of the technique is the requirement on the boundary parameters to lie in the region where the boundary breathers exist. Otherwise, strictly-speaking we could deduce nothing about the reflection factor since the comparison we have utilised would not be valid. However, it is perhaps worth noting that in the limit where $a_{0}=a_{1} \rightarrow 1 / 2$, the result does agree with the earlier conjectures of Ghoshal and Zamolodchikov concerning the Neumann boundary conditions despite the obvious fact that the Neumann boundary condition does not support boundary bound states.

\section{Weak-strong coupling duality}

We mentioned that the S-matrix describing the scattering of the sinh-Gordon particle is invariant under the exchange $\beta \rightarrow 4 \pi / \beta$. It is interesting to notice that there is a sense in which this duality extends to the reflection factors also. Consider the triple of coupling constants $\left(\beta, a_{0}, a_{1}\right)$.
If we define a new triple by making the change

$$
\left(\beta, a_{0}, a_{1}\right) \rightarrow \frac{4 \pi}{\beta^{2}}\left(\beta, a_{0}, a_{1}\right)
$$

then it is simple to check that the reflection factor

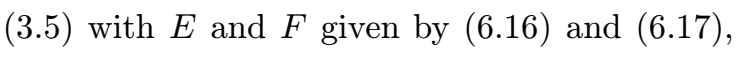
respectively, is invariant.

\section{Discussion and conclusions}

The sinh-Gordon model is just about the simplest massive model one might contemplate beyond free field theory. Yet it has been a long and tortuous road to discover a dynamical argument which would enable us to derive a relationship between the parameters occurring in the Lagrangian formulation of the model and the parameters arising from other, purely algebraic considerations - in the sense of deductions made using the boundary version of the Yang-Baxter equations together with the bootstrap. Our argument is certainly non-perturbative but it might not be exact. We hope that it will turn out to be exact but we cannot be sure. ${ }^{2}$

The properties of the boundary breathers we have found are quite intriguing. We should like to see an explanation for the surprising facts, for example concerning the dependence of the classical action on the boundary parameters. We would also be interested in exploring the situation with two boundaries. There, the field theory is confined to an interval and the spectrum of states within the interval should depend on the bulk coupling and four boundary parameters. One might expect the energy spectrum to be determined by a pair of reflection factors, one for each boundary, but that idea requires the assumption of factorization. In other words, if the boundaries behave independently we would expect a relationship of the type

$$
e^{4 i k L} R^{(L)}(k) R^{(-L)}(-k)=1,
$$

where the two boundaries are situated at $x=$ $\pm L$, and the corresponding reflection factors are $R^{( \pm L)}$. It would be interesting to see how this

\footnotetext{
${ }^{2}$ It should be remarked that Al. Zamolodchikov has also calculated (but not yet published) the parameter dependence derived using quite different arguments [닌.
} 
spectrum might be compatible with the quantization of periodic classical solutions.

There are several directions to go beyond sinh-Gordon. For example, the next simplest model with a single scalar field is the model based on $a_{2}^{(2)}$ data. There, instead of having two boundary parameters, there is only one but it can arise in two distinct ways in]. For this reason, we expect a greater variety of boundary breathers. The classical reflection factors are known [i $\left.\overline{1}_{1}^{1}\right]$ but at the moment the analogue of Ghoshal's formula has not been found. Indeed, analysing the boundary breathers might be of great assistance for this case, to serve as a guide in finding the correct expressions. Besides, there is a lot to do to classify completely all the reflection data for all the affine Toda models, extending what is already known for the $a_{n}^{(1)}$ series $\left[{ }_{1}^{1} 4_{1}^{\prime}\right]$.

\section{Acknowledgments}

I am grateful to my co-workers Medina Ablikim, Alireza Chenaghlou, Gustav Delius, Uli Harder, Viktoria Malyshenko and Anne Taormina for enjoyable collaborations, to the Universities of Lyon, Mons, Montpellier and Trieste for their hospitality at various times, and to the European Commission for financial support under the Training and Mobility of Researchers Network Contract ERBFMRX-CT-960012.

\section{References}

[1] M. Ameduri, R. Konik and A. LeClair Boundary sine-Gordon interactions at the free fermion point Phys. Letts. B354 (1995) 376; hep-th/9503088

[2] P. Bowcock, E. Corrigan, P. E. Dorey and R. H. Rietdijk, Classically integrable boundary conditions for affine Toda field theories, Nucl. Phys. B445 (1995) 469; 'hep-th/9501098;

[3] H.W. Braden, E. Corrigan, P.E. Dorey and R. Sasaki, Affine Toda field theory and exact $S$ matrices, Nucl. Phys. B3381990 689.

[4] A. Chenaghlou and E. Corrigan, First order quantum corrections to the classical reflection factor of the sinh-Gordon model, to appear in Int. J. Mod. Phys. A; ihep-th/0002065.'
[5] A. Chenaghlou, On the quantum reflection factor for the sinh-Gordon model with general boundary conditions, to appear in Int. J. Mod. Phys. A; hep-th/0004121.

[6] E. Corrigan, Integrable field theory with boundary conditions, in Frontiers in Quantum Field Theory eds. Chao-Zheng Zha and Ke Wu (World Scientific 1998); hep-th/9612138.

[7] E. Corrigan, On duality and reflection factors for the sinh-Gordon model with a boundary, Int. J. Mod. Phys A13 (1998) 2709; hep-th/9707235.

[8] E. Corrigan and G Delius, Boundary breathers in the sinh-Gordon model, J. Phys. A32 (1999) 8001-14; 'hep-th/9909145!'

[9] E. Corrigan, P.E. Dorey, R.H. Rietdijk and R. Sasaki, Affine Toda field theory on a half line, Phys. Letts. B333199483; 'hep-th/9404108'.

[10] E. Corrigan, P.E. Dorey and R.H. Rietdijk, Aspects of affine Toda field theory on a halfline, Prog. Theor. Phys. Suppl. 118 (1995) 143; hep-th/9407148.

[11] E. Corrigan and U.K.F. Harder, unpublished; U.K.F. Harder, PhD Thesis, University of Durham 1996.

[12] E. Corrigan and A. Taormina, Reflection factors and a two-parameter family of boundary bound states in the sinh-Gordon model, submitted to J. Phys. A; hep-th/0008237!

[13] R.F. Dashen, B. Hasslacher and A. Neveu, The particle spectrum in model field theories from semi-classical functional integral techniques, Phys. Rev. D11 (1975) 3424.

[14] G.W. Delius and G.M. Gandenberger, Particle reflection amplitudes in $a_{n}^{(1)}$ Toda field theories, Nucl. Phys. B554 (1999) 325; 'hep-th/9904002.

[15] L.D. Faddeev and V.E. Korepin, Quantum theory of solitons, Phys. Rep. 42 (1978) 1-87.

[16] A. Fring and R. Köberle, Affine Toda field theory in the presence of reflecting boundaries, Nucl. Phys. B419 (1994) 647; hep-th/9309142.

[17] A. Fring and R. Köberle, Boundary bound states in affine Toda field theories, Int. J. Mod. Phys. A10 (1995) 739; hep-th/9404188'.

[18] S. Ghoshal and A. Zamolodchikov, Boundary SMatrix and Boundary State in Two Dimensional Integrable Field Theory, Int. Jour. Mod. Phys. A9 (1994), 3841; hep-th/9306002. 
[19] S. Ghoshal, Bound State Boundary S-Matrix of the Sine-Gordon Model, Int. J. Mod. Phys. A9 (1994), 4801; hep-th/9310188',

[20] J.D. Kim Boundary reflection matrix in perturbative quantum field theory Phys. Letts. B353 (1995) 213; hep-th/9504016

[21] A.H. Luther, Quantum solitons in Statistical Physics, Solitons, eds R.K. Bullough and P.J. Caudrey, Topics in Current Physics 17 (Springer-Verlag 1980) 355.

[22] R. Rajaraman, Solitons and Instantons (North Holland 1982).

[23] N. Topor, Perturbation method for boundary $S$ matrix in $2 D$ quantum field theory, Mod. Phys. Lett. A12 (1997) 2951.

[24] Al. B. Zamolodchikov, private communication. 